\title{
Staphylococcus aureus Bacteremia Risk in Hemodialysis Patients Using the Buttonhole Cannulation Technique: A Prospective Multicenter Study
}

\author{
Rie Glerup, My Svensson, Jens D. Jensen, and Jeppe H. Christensen
}

Rationale \& Objective: Arteriovenous fistulas are the preferred access type for hemodialysis. The buttonhole needling technique has become an alternative to stepladder or area puncture. However, an increased risk for infection has been described. The present study examined the risk for infectious complications with different needling techniques.

Study Design: Prospective multicenter observational cohort study with 5 years of follow-up.

Setting \& Participants: In-center hemodialysis patients from 5 hemodialysis units in Denmark, dialyzed on a native arteriovenous fistula. $286 \mathrm{pa}$ tients were included; 144 cannulated with the buttonhole technique.

Exposure: The buttonhole cannulation technique was compared to the stepladder or area puncture technique.

Outcomes: Primary end points: event rates of access-related Staphylococcus aureus bacteremia and the HR for first access-related $S$ aureus bacteremia. Secondary end points: local infections and access-related $S$ aureus bacteremia-related metastatic infections and mortality.

Analytical Approach: Time-to-event analysis using Cox proportional hazards regression to estimate the HR of access-related $S$ aureus bacteremia in buttonhole cannulation compared to stepladder/ area puncture. Poisson regression was used for incidence rate ratio calculations.

Results: $S$ aureus caused 48 access-related bacteremias; $43(90 \%)$ in the buttonhole group compared with $5(10 \%)$ in the stepladder/area group. The HR for first access-related $S$ aureus bacteremia was significantly higher for buttonhole cannulation compared to stepladder/area needling (unadjusted, 6.8 [95\% Cl, 2.4-19.1]; adjusted, 8.4 [95\% Cl, 2.9-24.2]). The incidence rate ratio for access-related $S$ aureus bacteremia was $6.8(95 \% \mathrm{Cl}, 2.9-16.1)$, and the incidence rate ratio of local cannulation-site infection without access-related $S$ aureus bacteremia was $3.8(95 \% \mathrm{Cl}, 1.3-15.4)$ for buttonhole cannulation compared to stepladder/area needling.

Limitations: Nonrandomized observational design, prevalent hemodialysis patients.

Conclusions: Access-related $S$ aureus bacteremia rates were very high for buttonhole cannulation compared to stepladder/area needling, questioning the use of buttonhole cannulation in routine clinical practice. A restrictive approach to buttonhole use is recommended, with buttonhole cannulation only being used as a second alternative to area technique when stepladder cannulation is not feasible.
Complete author and article information provided before references.

Correspondence to R. Glerup (rig@rn.dk)

Kidney Med. 1(5):263-270.

Published online

September 11, 2019.

doi: 10.1016/

j.xkme.2019.07.007

(C) 2019 The Authors.

Published by Elsevier Inc. on behalf of the National Kidney Foundation, Inc. This is an open access article under the CC BY-NC-ND license (http://

creativecommons.org/ licenses/by-nc-nd/4.0/).
A rteriovenous fistulas (AVFs), when they are functional, demonstrate longer patency and fewer infectious complications compared with central venous catheters (CVCs) and arteriovenous grafts. ${ }^{1}$ As a consequence, AVF is the recommended vascular access for patients treated with

\section{Editorial, p. 235}

long-term hemodialysis (HD). ${ }^{2,3}$

Cannulation of the AVF uses 1 of 2 methods $^{4}$ : either stepladder or area technique, in which the site for needle insertion is changed for each HD session, or buttonhole cannulation, in which the site of needle insertion is kept constant. Buttonhole cannulation creates a fibrous tunnel between the skin and the vessel lumen over time, allowing the use of blunt needles to access the fistula. The buttonhole technique was originally used for demanding fistulas with short segments suitable for puncture, but now its use has been extended to a wider range of HD patients; both in-center HD and home HD (HHD) patients.
Possible advantages of buttonhole cannulation, such as longer AVF patency, fewer AVF interventions, and reduced cannulation pain, have not been convincingly documented because study results are inconsistent, ${ }^{5-8}$ and instead, increasing evidence suggests that buttonhole cannulation increases the risk for infection. ${ }^{6-10}$ A few randomized controlled trials (RCTs) have been published with a limited number of patients and short follow-up ${ }^{8,11-14}$; they reported very low infection rates in both groups. Most observational studies addressing the issue are retrospective and small, with short follow-up and lack of a proper control group, but the trend is toward a higher incidence of AVF-related infections in buttonhole cannulation compared to the stepladder/area cannulation technique.

Staphylococcus aureus is the leading cause of bacteremia in HD patients ${ }^{15}$ and is associated with severe complications such as endocarditis and osteomyelitis. The pathogen is the main cause of infectious morbidity related to vascular access in HD patients ${ }^{1}$ and often causes patient death. ${ }^{16}$ It has the ability to colonize a large part of the HD population 
without signs of disease but also to cause fulminant host invasions.

The aim of this multicenter study was to compare the risk for access-related $S$ aureus infection in HD patients cannulated using the buttonhole technique with patients cannulated using the stepladder/area technique.

\section{METHODS}

\section{Study Design}

We performed a prospective observational cohort study with 5 years of follow-up enrolling patients during a 4month period (December 2010 to March 2011). Patients receiving $\mathrm{HD}$ at 5 different $\mathrm{HD}$ facilities in Jutland, Denmark (Aalborg, Aarhus, Hjoerring, Randers, and Horsens), were assessed for eligibility. The number of patients receiving $\mathrm{HD}$ in these facilities determined the sample size.

\section{Inclusion/Exclusion Criteria}

Inclusion criteria were age older than 18 years and undergoing maintenance treatment with in-center HD using a native AVF. Exclusion criteria were presumed inability to understand informed consent and acute kidney failure.

\section{Data Collection}

After informed consent was obtained, patients underwent physical examination, blood sampling, and nasal swabbing. Blood samples were drawn from both a peripheral vein and the arterial needle before the start of a dialysis session. In all participants, medical history was obtained from both patient interview and medical charts at inclusion.

One physician (R.G.) reviewed all medical records at study entry and during follow-up. Patients were followed up for 5 years or censored in case of death, loss to followup, kidney transplantation, change to HHD or peritoneal dialysis, or permanent change of vascular access to arteriovenous graft or tunneled CVC. Clinical events were recorded; cause and length of any hospital admission and time and type of change in dialysis access and puncture technique (stepladder/area cannulation or buttonhole technique). For access-related $S$ aureus bacteremia, metastatic complications were recorded. Details of all accessrelated infections requiring hospitalization were collected.

\section{Outcomes}

The primary outcome was access-related $S$ aureus bacteremia. Blood specimens for culture were routinely obtained in case of fever or when bacteremia was suspected for other reasons, and access-related $S$ aureus bacteremia was defined as 1 or more positive blood culture with $S$ aureus and absence of a source of infection other than the AVF. Two successive bacteremias in a patient were regarded as separate events when the second bacteremia occurred more than 3 weeks after cessation of antibiotic treatment. Survival analysis with time to first access-related $S$ aureus bacteremia as the outcome measure was used to compare the risk for access-related $S$ aureus bacteremia with buttonhole cannulation versus stepladder/area fistula needling. Because cannulation techniques and vascular accesses changed for several patients during follow-up, data were split at the time of each change for the individual patients in the final Cox regression analysis, allowing each patient to add event-free time to the new cannulation method. In the Cox regression analysis, patients were censored at time of first event, disregarding follow-up time and events hereafter. For calculation of risk rates of infection, participation time was calculated as the sum of all AVF days for each patient according to cannulation technique; time receiving HD with a CVC was excluded.

The secondary outcome of local cannulation-site infection requiring hospital admission was defined as admission to the hospital with erythema, pain, warmth, swelling, or discharge from the cannulation site deemed by the treating clinicians to represent a local infection.

\section{Cannulation Practices}

All $5 \mathrm{HD}$ facilities participating in the study offered both buttonhole and stepladder/area cannulation technique to HD patients. The choice of cannulation method used in a given patient was made on the basis of a combination of patient preference, nurse preferences, and facility trends. There were no absolute contraindications for buttonhole tract creation, but stepladder/area technique was often favored in patients receiving HD fewer than 3 times per week or in patients with previous endocarditis and/or artificial heart valves. Buttonholes were preferred for patients preparing for HHD. All staff performing AVF cannulation were registered nurses. In a given patient, only 1 to 2 nurses were cannulating during buttonhole tract creation, but in established buttonholes, no such restriction was enforced.

\section{Ethics}

The study protocol was approved by the Regional Research Ethics Committee of The North Jutland Region (protocol $\mathrm{N}-20100041$ ) and was conducted in accordance with the ethical principles of the Declaration of Helsinki.

\section{Biochemical Analyses}

Blood cultures from both peripheral vein and dialysis access were incubated and microbial growth was detected using the BacT/ALERT system (BioMérieux). When blood cultures flagged positive on the system, aliquots of the blood culture medium were used to identify the cultured organism. An S aureus isolate from each $S$ aureus bacteremia was referred to the national reference laboratory (Statens Serum Institut, Copenhagen, Denmark). Nasal swabs were tested for $\mathrm{S}$ aureus using routine methods. C-Reactive protein was measured using the Tina-quant CRP Gen. 3 ultrasensitive particle-enhanced immunoturbidimetric assay (Roche Diagnostics GmbH). 


\section{Statistical Analyses}

Baseline data are given as mean \pm standard deviation, median with interquartile range, or percentage, as appropriate.

Time to first access-related $S$ aureus bacteremia was examined using Kaplan-Meier and Cox proportional hazards approaches disregarding follow-up time after the first event. To investigate whether observed differences between groups were confounded by demographic or clinical factors, the following variables were added to the Cox model: age, sex, $S$ aureus nasal carriage, and whether there were any changes in cannulation technique. To test the proportional hazards assumption, we used Schoenfeld residuals and log-log plots.

We calculated the person-time incidence rate as the number of events per total number of fistula-days (standardized to 1,000 fistula-days). Comparison of event rates between groups was done using Poisson regression with clustered sandwich estimation of standard errors, allowing for correlation within each patient for recurrent events. To the Poisson regressions, the binary variable of change in cannulation technique was added. Difference in length of access-related $S$ aureus bacteremia-caused hospitals stays was estimated using ordinary linear regression. Confidence intervals (CIs) and P values were calculated using bootstrap with 10,000 replications to accommodate non-normality. Quantitative variables were handled as continuous.

Statistical analysis was performed using STATA (version 14 MP; Stata Corp), and the level of statistical significance was set at $5 \%$.

\section{RESULTS}

\section{Study Participants}

A total of 415 patients were receiving HD in the 5 centers at the time of inclusion, and 286 patients were finally included (Fig 1); 144 cannulated using the buttonhole technique, and 142, using stepladder/area needling.

All changes in cannulation technique were registered and in total there were 205 periods with buttonhole cannulation and 193 periods with stepladder/area cannulation. Stepladder/area cannulation was used at some point during follow-up for 173 patients, and buttonhole cannulation was used for 183 patients at some point (Fig S1). The reasons for cannulation change were local infection, access-related $S$ aureus bacteremia, difficult cannulations, cannulation pain, patient preference, staff preference, long bleeding time after HD, and preparation for HHD.

Total follow-up time was 803 patient-years, 1,025 days per participant (1,012 days excluding temporary acute or tunneled $\mathrm{CVCs}$ ). Mean follow-up time per period in the buttonhole group was 783 (range, 4-1,858) days, and in the stepladder/area group, 667 (range, 4-1,857) days. (Censoring causes are given in Fig S2).

Participants in the buttonhole needling group were younger with a slightly longer HD vintage compared with

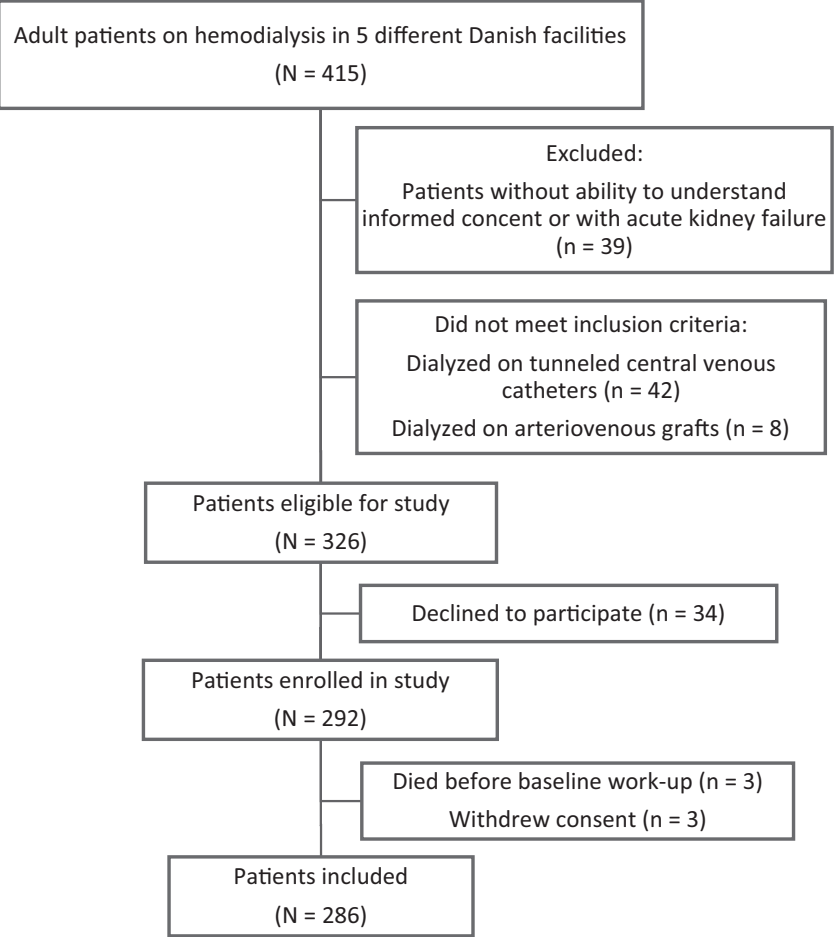

Figure 1. Patient flow diagram depicts enrollment.

stepladder/area group participants, and the HD frequency was higher in the buttonhole group, mainly driven by more patients in the stepladder/area group dialyzing only twice weekly. In the stepladder/area group, there were more men and more comorbid conditions (cancer and cardiovascular disease) compared with the buttonhole group (Table 1). Immunosuppressive therapy or signs of infection were similar, and $\mathrm{S}$ aureus nasal carriage did not differ between groups ( $40 \%$ and $43 \%$ ). A description of baseline characteristics with the cohort divided into 3 cannulation groups (buttonhole only, stepladder/area only, and patients who switched cannulation technique during follow-up) is found in Table S1. Ten participants in the buttonhole group and 2 in the stepladder/area group had positive blood cultures at baseline (Table 1). These positive cultures were regarded as contamination, and the involved patients showed no signs of bacteremia.

\section{$S$ aureus Bacteremias}

During follow-up, 168 events of positive blood cultures were found (cultured pathogens are given in Table S2). Thirty-six patients experienced 48 episodes of accessrelated S aureus bacteremia, all methicillin susceptible, and 32 of these patients were in the buttonhole group (Table 2). Men in the buttonhole group accounted for 33 of the 43 access-related $S$ aureus bacteremia episodes. Two of the 19 patients in the buttonhole group and none of the 4 patients in the stepladder/area group performing selfcannulation developed access-related $S$ aureus bacteremia. In total, 18 patients (16 men) developed access-related $S$ aureus bacteremia during the first year; 16 in the buttonhole 
Table 1. Baseline Demographic Characteristics by Puncture Technique at Baseline

\begin{tabular}{|c|c|c|}
\hline Characteristics & $\begin{array}{l}\text { Buttonhole Puncture } \\
\text { at Baseline }(N=144)\end{array}$ & $\begin{array}{l}\text { Sharp Needle } \\
\text { at Baseline } \\
(\mathrm{N}=142)\end{array}$ \\
\hline Age, y & $63.7(50.6-74.2)$ & $\begin{array}{l}71.2(59.3- \\
79.2)\end{array}$ \\
\hline Age $>65$ y & $67(47 \%)$ & $91(64 \%)$ \\
\hline Male sex & $86(60 \%)$ & $104(73 \%)$ \\
\hline \multicolumn{3}{|l|}{ Diabetes } \\
\hline No & $109(76 \%)$ & $104(73 \%)$ \\
\hline Type 1 & $12(8 \%)$ & $7(5 \%)$ \\
\hline Type 2 & $23(16 \%)$ & $31(22 \%)$ \\
\hline \multicolumn{3}{|l|}{ Tobacco use } \\
\hline Never & $51(35 \%)$ & $42(30 \%)$ \\
\hline Previous & $52(36 \%)$ & $66(46 \%)$ \\
\hline Active & $40(28 \%)$ & $33(23 \%)$ \\
\hline \multicolumn{3}{|l|}{ Cause of ESRD } \\
\hline Diabetic nephropathy & $25(17 \%)$ & $24(17 \%)$ \\
\hline Glomerulonephritis & $24(17 \%)$ & $13(9 \%)$ \\
\hline Vasculitis & $10(7 \%)$ & $4(3 \%)$ \\
\hline $\begin{array}{l}\text { Hypertension and } \\
\text { vascular }\end{array}$ & $15(10 \%)$ & $25(18 \%)$ \\
\hline $\begin{array}{l}\text { Polycystic kidney } \\
\text { disease }\end{array}$ & $17(12 \%)$ & $12(9 \%)$ \\
\hline Uropathy & $7(5 \%)$ & $14(10 \%)$ \\
\hline Other & $15(10 \%)$ & $25(18 \%)$ \\
\hline Unknown & $31(22 \%)$ & $25(18 \%)$ \\
\hline $\begin{array}{l}\text { Previous cardiovascular } \\
\text { disease }\end{array}$ & $60(42 \%)$ & $77(54 \%)$ \\
\hline TTRRT, y & $3.9(2.0-8.4)$ & $3.4(1.0-8.8)$ \\
\hline HD vintage, $y$ & $2.8(1.7-6.0)$ & $2.5(0.8-5.5)$ \\
\hline \multicolumn{3}{|l|}{ Vascular access: } \\
\hline AVF ante brachium & $113(78 \%)$ & $111(78 \%)$ \\
\hline AVF brachium & $30(21 \%)$ & $31(22 \%)$ \\
\hline AVF femur & $1(0.7 \%)$ & $0(0 \%)$ \\
\hline Self-cannulation & $19(13 \%)$ & $4(3 \%)$ \\
\hline Access vintage, $y$ & $3.0(1.5-6.0)$ & $2.3(1.0-5.2)$ \\
\hline \multicolumn{3}{|l|}{ Dialysis modality } \\
\hline $\mathrm{HD}$ & $119(83 \%)$ & $121(85 \%)$ \\
\hline HDF & $25(17 \%)$ & $21(15 \%)$ \\
\hline \multicolumn{3}{|l|}{ HD filter } \\
\hline High flux & $102(71 \%)$ & $76(54 \%)$ \\
\hline Low flux & $42(29 \%)$ & $66(46 \%)$ \\
\hline \multicolumn{3}{|l|}{$\begin{array}{l}\text { HD frequency, } \\
\text { sessions/wk }\end{array}$} \\
\hline$<3$ & $13(9 \%)$ & $36(27 \%)$ \\
\hline 3 & $117(81 \%)$ & $95(67 \%)$ \\
\hline$>3$ & $14(10 \%)$ & $11(8 \%)$ \\
\hline $\begin{array}{l}\text { Systolic blood } \\
\text { pressure, mm Hg }\end{array}$ & $144 \pm 26$ & $141 \pm 24$ \\
\hline $\begin{array}{l}\text { Diastolic blood } \\
\text { pressure, } \mathrm{mm} \mathrm{Hg}\end{array}$ & $73 \pm 15$ & $69 \pm 15$ \\
\hline Statin treatment & $44(31 \%)$ & $52(37 \%)$ \\
\hline $\begin{array}{l}\text { Erythropoietin } \\
\text { treatment }\end{array}$ & $137(95 \%)$ & $124(87 \%)$ \\
\hline Iron treatment & $117(81 \%)$ & $107(75 \%)$ \\
\hline $\begin{array}{l}\text { Immunosuppressive } \\
\text { treatment }\end{array}$ & $12(8 \%)$ & $12(9 \%)$ \\
\hline
\end{tabular}

(Continued)
Table 1 (Cont'd). Baseline Demographic Characteristics by Puncture Technique at Baseline

\begin{tabular}{|c|c|c|}
\hline Characteristics & $\begin{array}{l}\text { Buttonhole Puncture } \\
\text { at Baseline }(N=144)\end{array}$ & $\begin{array}{l}\text { Sharp Needle } \\
\text { at Baseline } \\
(\mathrm{N}=142)\end{array}$ \\
\hline $\begin{array}{l}\text { Immunosuppressive } \\
\text { last } 6 \text { mo }\end{array}$ & $5(4 \%)$ & $1(0.7 \%)$ \\
\hline $\begin{array}{l}\text { Active cancer at } \\
\text { inclusion }^{\mathrm{a}}\end{array}$ & $5(4 \%)$ & $14(10 \%)$ \\
\hline Previous cancer ${ }^{a}$ & $14(10 \%)$ & $18(13 \%)$ \\
\hline $\begin{array}{l}\text { C-Reactive protein, } \\
\mathrm{mg} / \mathrm{L}\end{array}$ & $5.6(1.7-12.5)$ & $6.0(2.8-16.5)$ \\
\hline Antibiotics at inclusion & $15(10 \%)$ & $9(6 \%)$ \\
\hline Antibiotics last mo & $18(13 \%)$ & $23(16 \%)$ \\
\hline $\begin{array}{l}\text { Subjective sign of } \\
\text { infection }\end{array}$ & $28(19 \%)$ & $21(15 \%)$ \\
\hline $\begin{array}{l}\text { Objective signs of } \\
\text { infection }\end{array}$ & $12(8 \%)$ & $15(11 \%)$ \\
\hline Positive nasal swab & $58(40 \%)$ & $61(43 \%)$ \\
\hline $\begin{array}{l}\text { Positive blood culture, } \\
\text { all }\end{array}$ & $10(7 \%)$ & $2(1 \%)$ \\
\hline $\begin{array}{l}\text { Positive blood culture } \\
\text { from peripheral vein }\end{array}$ & $1(0.7 \%)$ & $0(0 \%)$ \\
\hline $\begin{array}{l}\text { Blood culture with } \\
\text { CoNS }\end{array}$ & $8(6 \%)$ & $2(1 \%)$ \\
\hline $\begin{array}{l}\text { Blood culture with } \\
\text { Staphylococcus aureus }\end{array}$ & $2(1 \%)$ & $0(0 \%)$ \\
\hline \multicolumn{3}{|c|}{$\begin{array}{l}\text { Note: Values expressed as mean } \pm \text { standard deviation, median (range, 25th- } \\
\text { 75th percentiles), or number (percent). } \\
\text { Abbreviations: AVF, arteriovenous fistula; CoNS, coagulase-negative staphy- } \\
\text { lococci; ESRD, end-stage renal disease; HD, hemodialysis; HDF, hemodiafil- } \\
\text { tration; TTRRT, total time on renal replacement therapy. } \\
\text { aExcept basal cell carcinoma and squamous cell carcinoma of the skin. }\end{array}$} \\
\hline
\end{tabular}

group. In the buttonhole group, 69\% of patients with access-related $S$ aureus bacteremia during the first year of follow-up were $S$ aureus nasal carriers at baseline, compared to none in the stepladder/area group. The unadjusted hazard ratio (HR) for first access-related $S$ aureus bacteremia for a baseline nasal carrier was 2.5 (95\% CI, 1.3-4.9) compared to a non-nasal carrier. Looking only at the buttonhole patients, the HR was 3.0 (95\% CI, 1.4-6.1).

The person-time incidence rate of access-related $S$ aureus bacteremia was 0.268 per 1,000 fistula-days in the buttonhole group compared to 0.0388 in the stepladder/ area group (Table 2), giving an incidence rate ratio (IRR) of 6.78 (95\% CI, 2.85-16.14). The time to first accessrelated $S$ aureus bacteremia was significantly longer in the stepladder/area group compared with the buttonhole group (Fig 2). The HR for first access-related $S$ aureus bacteremia is shown in Table 3. The addition of HD facility to the model increased the HR of access-related $S$ aureus bacteremia further (HR, 10.1 [95\% CI, 3.5-29.4]). The addition of HD frequency to the model did not alter the coefficient of interest.

\section{S aureus Bacteremia Complications and Local Infections}

Access-related S aureus bacteremias caused 742 in-hospital days in the buttonhole group and 89 days in the 
Table 2. Infectious Complications by Cannulation Technique

\begin{tabular}{|c|c|c|c|c|c|c|}
\hline & \multicolumn{2}{|c|}{$\begin{array}{l}\text { Buttonhole } \\
(160,556 \\
\text { fistula-d) }\end{array}$} & \multicolumn{2}{|c|}{$\begin{array}{l}\text { Stepladder or } \\
\text { Area }(128,806 \\
\text { fistula-d) }\end{array}$} & \multirow[b]{2}{*}{ IRR (95\% CI) } & \multirow[b]{2}{*}{$P$} \\
\hline & $\mathrm{N}$ & Rate $^{a}$ & $\bar{N}$ & Rate $^{a}$ & & \\
\hline Bacteremia events & 124 & 0.772 & 40 & 0.311 & $2.48(1.60-3.84)$ & $<0.001$ \\
\hline $\begin{array}{l}\text { Access-related Staphylococcus aureus } \\
\text { bacteremia }\end{array}$ & 43 & 0.268 & 5 & 0.0388 & $6.78(2.85-16.14)$ & $<0.001$ \\
\hline All staphylococci & 82 & 0.511 & 15 & 0.117 & $4.33(2.39-7.86)$ & $<0.001$ \\
\hline 1st access-related $S$ aureus bacteremia & 32 & 0.199 & 4 & 0.0311 & $6.46(2.31-18.05)$ & $<0.001$ \\
\hline $\begin{array}{l}\text { Non-access-related } S \text { aureus bacteremia } \\
\text { cannulation-site infections requiring admission }\end{array}$ & 19 & 0.118 & 4 & 0.0311 & $3.91(1.35-11.34)$ & 0.01 \\
\hline $\begin{array}{l}\text { Combined access-related } S \text { aureus bacteremia } \\
\text { and cannulation-site infection }\end{array}$ & 7 & 0.0436 & 1 & 0.00776 & $5.58(0.68-45.54)$ & 0.11 \\
\hline $\begin{array}{l}\text { Metastatic access-related } S \text { aureus bacteremia } \\
\text { infections }\end{array}$ & 8 & 0.0498 & 1 & 0.00776 & $6.46(0.80-51.97)$ & 0.08 \\
\hline Total no. of infectious endocarditis & 12 & 0.0747 & 2 & 0.0155 & $4.96(1.11-22.23)$ & 0.04 \\
\hline
\end{tabular}

Abbreviations: $\mathrm{Cl}$, confidence interval; IRR, incidence rate ratio.

aPerson-time incidence rate per 1,000 fistula-days.

stepladder/area group, mean difference per stay was 2.04 (95\% CI, 0.83-3.25) days longer in the buttonhole group, and local cannulation-site infection without access-related $S$ aureus bacteremia caused 19 admissions in 16 patients in the buttonhole group in contrast to 4 admissions in the stepladder/area group (IRR, 3.91 [95\% CI, 1.35-11.34]). Eight buttonhole patients with access-related $S$ aureus bacteremia developed metastatic infections, mainly endocarditis $(n=7)$. In comparison, 1 case of endocarditis was observed in the stepladder/area group with access-related $S$ aureus bacteremia. Two of $32(6.3 \%)$ patients in the buttonhole group and 1 of 4 (25\%) in the stepladder/area group died within 30 days of first access-related $S$ aureus bacteremia.

The IRR of recurrent access-related $S$ aureus bacteremia for AVF users was 3.2 (95\% CI, 1.5-6.3) compared to first access-related $S$ aureus bacteremia.

\section{DISCUSSION}

A functioning vascular access is regarded as the lifeline for the HD patient. AVFs are the preferred access type due to fewer complications compared with other types of vascular accesses, but whether sharp or blunt needles should be preferred is still unclear. Our study clearly shows that the risk for infection, especially access-related $\mathrm{S}$ aureus bacteremia, is significantly increased using the buttonhole technique compared to the stepladder/area technique. This relatively large cohort with 5-year followup is one of the largest studies examining infectious outcomes of buttonhole cannulation. With an adjusted HR $>8$ for the first access-related $S$ aureus bacteremia and an access-related $S$ aureus bacteremia rate ratio of 6.8 for buttonhole compared to stepladder/area technique, our study confirms the results from prior observational studies.

Previous studies have examined the incidence of $S$ aureus bacteremia after switching from rope-ladder technique to

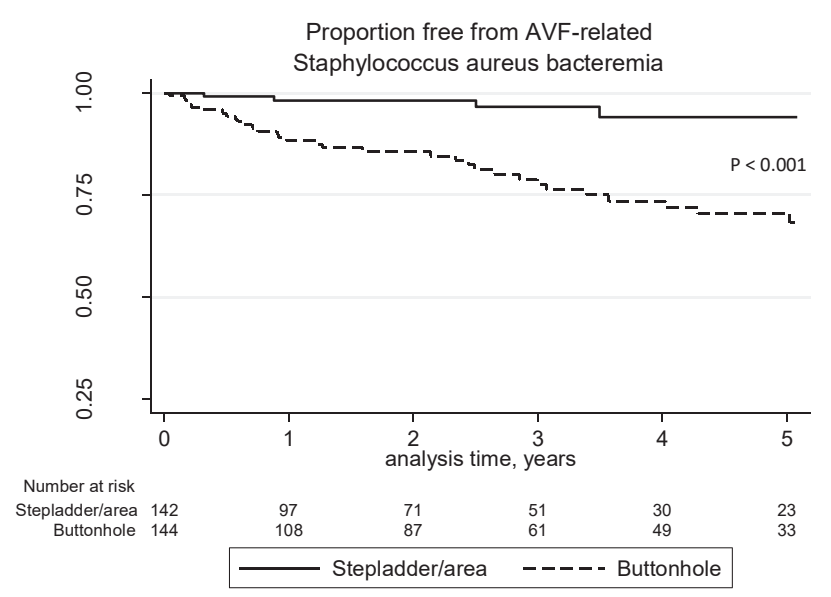

Figure 2. Kaplan-Meyer survival estimates. Abbreviation: AVF, arteriovenous fistula.

buttonhole technique. In line with our results, Labriola et $\mathrm{al}^{17}$ in a single-center study retrospectively observed a nearly 7 -fold increase in the incidence of access-related $S$ aureus bacteremia after a change from rope-ladder technique to buttonhole technique in 177 in-center HD patients followed up for 510 patient-years. In contrast, Béchade et $\mathrm{al}^{18}$ evaluated patients from a low-care dialysis unit before and after a progressive switch from rope-ladder to buttonhole cannulation. The reported access-related $S$ aureus bacteremia rate was only $0.05 / 1,000$ patient-days in 115 patients followed up for 268 patient-years after conversion to buttonhole technique. The IRR after/before was 2.9. However, this small low-care unit with 6 patients dialyzing at a time and 1 attending nurse, with some patients performing selfcannulation, is not comparable to busy larger units like ours.

In a study from London, ${ }^{19}$ a large cohort of in-center HD patients was compared retrospectively for differences in infectious complications between cannulation techniques (and CVCs). Twelve episodes of access-related $S$ 
Table 3. HR for Developing Staphylococcus aureus Bacteremia Related to the Arteriovenous Fistula for Patients Using Buttonhole Technique or a Sharp Needle for Area/Stepladder Puncture

\begin{tabular}{llll}
\hline & HR & 95\% Cl & $P$ \\
\hline $\begin{array}{l}\text { Sharp needle for area or } \\
\text { stepladder puncture }\end{array}$ & Reference & & \\
\hline $\begin{array}{l}\text { Buttonhole technique } \\
\text { (unadjusted) }\end{array}$ & 6.76 & $2.39-19.13$ & $<0.001$ \\
\hline (adjusted) & 8.41 & $2.93-24.15$ & $<0.001$ \\
\hline
\end{tabular}

Note: Unadjusted and adjusted for age, sex, Staphylococcus aureus nasal carriage, and change of cannulation method during follow-up.

Abbreviations: $\mathrm{Cl}$, confidence interval; $\mathrm{HR}$, hazard ratio.

aureus bacteremia were observed in 219 buttonhole patients with 30 months of follow-up. The HR for developing access-related $S$ aureus bacteremia was 3.6 (95\% CI, $1.3-$ 9.6) compared with patients using area needling. All patients were screened every 3 months for nasal carriage of $S$ aureus and carriers were decolonized with chlorhexidine and/or mupirocin according to sensitivity. This eradication regimen may explain the lower rate of access-related $S$ aureus bacteremia compared with ours.

Four RCTs have reported on infectious outcomes in incenter HD patients randomly assigned to buttonhole or stepladder/area technique. ${ }^{11-14}$ In contrast to our results, all 4 RCTs reported low $S$ aureus bacteremia rates. One explanation could be short follow-up time in the RCTs, but another might be a protocol/Hawthorne effect affecting not only participants, but also members of the staff. ${ }^{20}$ Our study describes and reflects daily clinical practice. A larger RCT with long follow-up might help, but recruitment can be difficult, as seen in a recent pilot trial ${ }^{21}$ examining the feasibility of such a study in HHD patients. The authors succeeded in randomly assigning only 14 of 158 patients who started HHD training.

Many studies examining HHD patients report increased infection rates for the buttonhole technique compared to stepladder/area needling. ${ }^{6,22-24}$ Several factors may account for this variability in the published access-related $S$ aureus bacteremia rates. Differences in patient characteristics, especially when comparing in-center HD to HHD, are numerous; there is significant between-facility variability in access-related infection rates regardless of vascular access type and cannulation technique, ${ }^{25,26}$ possibly reflected in differences in clinical practices and expertise, as well as in differences in dialysis populations. Studies with short follow-up ${ }^{7,12-14}$ reported lower access-related $S$ aureus bacteremia rates, indicating that follow-up time is of importance and accounts for some of the reported variance.

It is important to recognize what causes the increased $S$ aureus bacteremia rate for the in-center HD buttonhole patients. Differences in patient characteristics and HD frequency could have an impact, but in our study, the risk for access-related $S$ aureus bacteremia was associated only with $S$ aureus nasal carriage.

In our study, $69 \%$ of patients in the buttonhole group who developed access-related $S$ aureus bacteremia during the first year of follow-up were $S$ aureus nasal carriers at baseline. This carrier status may play an important role in $S$ aureus bacteremia, and prophylactic approaches such as mupirocin applied to the buttonhole $\operatorname{site}^{27}$ or nasal mupirocin $^{28}$ may be promising, although concerns of potential mupirocin resistance must be considered. ${ }^{29,30} \mathrm{~S}$ aureus colonizing the nares may be autoinoculated on the skin overlying the cannulation site through the patient's hand. ${ }^{31}$ From the skin, buttonhole patients have a unique route to the bloodstream through the buttonhole tract. Sato et $\mathrm{al}^{32}$ found that a bulging deformity of the buttonhole entry site increased the risk for vascular access-related infection with an odds ratio of $5.4 \quad(\mathrm{P}=0.0085)$ compared with buttonholes with a flat entry. However, it is unknown whether this increased risk is caused by a more difficult scab removal or an expansion of the tract opening due to the bulging deformity.

A study of the bacteriology of the buttonhole cannulation tract recently showed that $38 \%$ of buttonhole cannulators had at least 1 positive culture result from the buttonhole tract or the cannula tip on 3 monthly sets of cultures and that $30 \%$ of those with positive tract culture also had concomitant bacteremia (based on cultures drawn from the access). ${ }^{33}$ During 9 months of follow-up, $60 \%$ of participants who had cultures with $S$ aureus or repeatedly Staphylococcus epidermidis-positive cultures developed accessrelated infections $(30 \%$ bacteremia and 30\% buttonhole infection) compared to infection in $6 \%$ of culture-negative participants. In the study by Nesrallah et al, ${ }^{27}$ they applied mupirocin ointment to the buttonhole entry site after an HD session, indicating that the application decolonized a buttonhole tract that was harboring $S$ aureus. Autoinoculation and possibly tract opening deformities may enable $S$ aureus to colonize the tract and when the equilibrium of host resistance and bacterial inoculum is upset, bacteremia may develop.

In our study, 30-day mortality after the first $S$ aureus bacteremia was relatively low in the buttonhole group $(6.3 \%)$. However, in the entire cohort, 30-day all-cause case mortality was $8.3 \%(3 / 36)$. In line with our results, Collier et a ${ }^{19}$ found a combined 30-day mortality rate of 9.7\% including patients using CVCs. In a nationwide Danish database study by Nielsen et $a l,{ }^{34}$ the 30 -day case fatality after $S$ aureus bacteremia among HD patients was $10.2 \%(95 \% \mathrm{CI}, 8.5 \%-12.1 \%)$ compared to $24.2 \%$ (95 CI, $21.2 \%-27.6 \%)$ among population controls. Outside Europe, reported mortality rates are higher, ranging from $20 \%$ (12-week mortality) after S aureus bacteremia in the United States ${ }^{16}$ to $46.9 \%$ (in-hospital mortality) in Japan. ${ }^{35}$ Thus, short-term mortality is lower in HD patients, especially those cannulating with the buttonhole technique, compared with population controls. This might be explained because the threshold for hospital admission and blood culturing of dialysis patients showing signs and symptoms compatible with $S$ aureus bacteremia may be lower in a hospital setting than in the general population. Thus, early initiation of correct therapy would result in 
improved survival compared with population controls. The low rate of methicillin-resistant $S$ aureus causing $S$ aureus bacteremia in Denmark also leads to early correct and effective antibiotic therapy. ${ }^{36}$

Wertheim et $\mathrm{al}^{37}$ showed that for nosocomial $S$ aureus bacteremia, nasal $S$ aureus carriers had a 3 -fold higher incidence (80\% endogenous) compared with noncarriers, but lower in-hospital $S$ aureus bacteremia-related mortality of $8 \%$ compared with $32 \%$ in noncarriers. This finding could not be explained by differences in age and underlying disease. An improved outcome in patients with $S$ aureus bacteremia caused by endogenous $S$ aureus strains indicate that the patient may have immunologically adapted to that particular strain, hence producing a more adequate immune response than noncarriers, or that exogenous strains might be more virulent. Disputing the thought of immunologic adaptation is the fact that several attempts to create an effective anti-S aureus vaccine have failed. ${ }^{38}$

Our study has several limitations. The observational design, though prospective, is inferior to a RCT. Patient characteristics, especially age, differed between cannulation groups. One would expect older patients to be at higher risk for developing S aureus bacteremia due to immune state and comorbid conditions. Buttonhole patients in our study were younger than participants in the stepladder/area group, thereby potentially underestimating the true HR. Between-center differences in protocol, preferences, and technique may strengthen the external validity of the study, but comparisons may be difficult to interpret. Our multicenter prospective cohort study had all data collected by the same investigator, thereby minimizing bias due to differences in data interpretation and admission coding.

In conclusion, we report the largest prospective multicenter cohort study of in-center HD patients comparing buttonhole cannulation to stepladder/area cannulation. Infection rates were almost 7 times higher in the buttonhole group, with an adjusted HR of time to first accessrelated $S$ aureus bacteremia of 8.4 , but mortality due to access-related $S$ aureus bacteremia was low. The data suggest that it might be advisable to identify and characterize the group of patients suitable for buttonhole cannulation to implement strict selection criteria. In our HD facilities, buttonhole and stepladder/area cannulation techniques have been equal first choices in in-center HD patients; however, the results question the use of buttonhole cannulation as a first choice. An individual restrictive attitude is recommended, with buttonhole as a second alternative to area technique when stepladder cannulation is not feasible.

\section{SUPPLEMENTARY MATERIAL}

\section{Supplementary File (PDF)}

Figure S1: Flowchart showing cannulation method switches

Figure S2: Flowchart showing causes for censoring

Table S1: Baseline characteristics by puncture technique during follow-up
Table S2: Cultured pathogens in positive blood cultures by puncture technique

\section{ARTICLE INFORMATION}

Authors' Full Names and Academic Degrees: Rie Glerup, MD, My Svensson, PhD, Jens D. Jensen, PhD, and Jeppe H. Christensen, DMSc.

Authors' Affiliations: Department of Nephrology, Aalborg University Hospital, Aalborg, Denmark (RG, JHC); Department of Renal Medicine, Akershus University Hospital, Lørenskog (MS); Institute of Clinical Medicine, University of Oslo, Oslo, Norway (MS); Department of Renal Medicine, Aarhus University Hospital (JDJ); Institute of Clinical Medicine, Aarhus University, Aarhus (JDJ); and Institute of Clinical Medicine, Aalborg University, Aalborg, Denmark (JHC).

Address for Correspondence: Rie Glerup, MD, Department of Nephrology, Aalborg University Hospital, Mølleparkvej 4, 9000 Aalborg, Denmark. E-mail: rig@rn.dk

Authors' Contributions: Research idea and study design: RG, MS, $\mathrm{JHC}$; data acquisition and analysis: RG; statistical analysis: RG; data interpretation: all authors; mentorship: MS, JDJ, JHC. Each author contributed important intellectual content during manuscript drafting and revision and accepts accountability for the overall work by ensuring that questions pertaining to the accuracy or integrity of any portion of the work are appropriately investigated and resolved.

Support: This work was supported by The Danish Kidney Society, The Danish Society of Nephrology, The North Denmark Region, Karen Elise Jensen's Foundation, The Hertha Christensen Foundation, The Helen and Ejnar Bjørnow Foundation, Medical specialist Heinrich Kopp's Grant, Lundbeck Foundation, The Spar Nord Foundation, and The Obel Family Foundation. The funders had no role in study design; collection, analysis, and interpretation of data; writing the report; or the decision to submit the report for publication.

Financial Disclosure: The authors declare that they have no relevant financial interests.

Acknowledgements: We thank the patients and HD units participating in this study. Henrik C. Schønheyder (Department of Clinical Microbiology, Aalborg University Hospital) rendered advice in an early phase of the study.

Peer Review: Received April 8, 2019. Evaluated by 3 external peer reviewers, with direct editorial input from the Statistical Editor and the Editor-in-Chief. Accepted in revised form July 30, 2019.

\section{REFERENCES}

1. Hoen B, Paul-Dauphin A, Hestin D, Kessler M. EPIBACDIAL: a multicenter prospective study of risk factors for bacteremia in chronic hemodialysis patients. J Am Soc Nephrol. 1998;9(5): 869-876.

2. National Kidney Foundation. KDOQI clinical practice guideline for vascular access. Am J Kidney Dis. 2006;48(suppl 1):S176S276.

3. Kumwenda M, Mitra S, Reid C. Clinical Practise Guideline Vascular Access for Haemodialysis - UK Renal Association. 6th ed. https://renal.org/wp-content/uploads/2017/06/vascularaccess.pdf. Accessed February 22, 2019.

4. Twardowski Z, Kubara H. Different sites versus constant sites of needle insertion into arteriovenous fistulas for treatment by repeated dialysis. Dial Transplant. 1979;8(10):978-980. 
5. Chan MR, Shobande O, Vats $\mathrm{H}$, et al. The effect of buttonhole cannulation vs. rope-ladder technique on hemodialysis access patency. Semin Dial. 2014;27(2):210-216.

6. Muir CA, Kotwal SS, Hawley CM, et al. Buttonhole cannulation and clinical outcomes in a home hemodialysis cohort and systematic review. Clin J Am Soc Nephrol. 2014;9(1):110-119.

7. van Loon MM, Goovaerts T, Kessels AG, van der Sande FM, Tordoir JH. Buttonhole needling of haemodialysis arteriovenous fistulae results in less complications and interventions compared to the rope-ladder technique. Nephrol Dial Transplant. 2010;25(1):225-230.

8. Chow J, Rayment G, San Miguel S, Gilbert M. A randomised controlled trial of buttonhole cannulation for the prevention of fistula access complications. J Ren Care. 2011;37(2):85-93.

9. Birchenough E, Moore C, Stevens K, Stewart S. Buttonhole cannulation in adult patients on hemodialysis: an increased risk of infection? Nephrol Nurs J. 2010;37(5):491-498, 555; quiz 499.

10. Ward F, Holian J, Watson A. Arteriovenous fistula buttonhole cannulation: early experience in an Irish haemodialysis unit [letter]. Nephrol Dial Transplant. 2011;26(11):3825; author reply 3825-3826.

11. Toma S, Shinzato $T$, Fukui $H$, et al. A timesaving method to create a fixed puncture route for the buttonhole technique. Nephrol Dial Transplant. 2003;18(10):2118-2121.

12. Struthers J, Allan A, Peel RK, Lambie SH. Buttonhole needling of ateriovenous fistulae: a randomized controlled trial. ASA/O J. 2010;56(4):319-322.

13. MacRae JM, Ahmed SB, Atkar R, Hemmelgarn BR. A randomized trial comparing buttonhole with rope ladder needling in conventional hemodialysis patients. Clin J Am Soc Nephrol. 2012;7(10):1632-1638.

14. Vaux E, King J, Lloyd S, et al. Effect of buttonhole cannulation with a polycarbonate PEG on in-center hemodialysis fistula outcomes: a randomized controlled trial. Am J Kidney Dis. 2013;62(1):81-88.

15. Vandecasteele SJ, Boelaert JR, De Vriese AS. Staphylococcus aureus infections in hemodialysis: what a nephrologist should know. Clin J Am Soc Nephrol. 2009;4(8):1388-1400.

16. Li Y, Friedman JY, O'Neal BF, et al. Outcomes of Staphylococcus aureus infection in hemodialysis-dependent patients. Clin J Am Soc Nephrol. 2009;4(2):428-434.

17. Labriola L, Crott R, Desmet C, Andre G, Jadoul M. Infectious complications following conversion to buttonhole cannulation of native arteriovenous fistulas: a quality improvement report. Am J Kidney Dis. 2011;57(3):442-448.

18. Béchade C, Goovaerts $T$, Cougnet $P$, Labriola L, Jadoul M, Goffin E. Buttonhole cannulation is not associated with more AVF infections in a low-care satellite dialysis unit: a long-term longitudinal study. PLoS One. 2015;10(11):e0142256.

19. Collier S, Kandil H, Yewnetu E, Cross J, Caplin B, Davenport A. Infection rates following buttonhole cannulation in hemodialysis patients. Ther Apher Dial. 2016;20(5):476-482.

20. Braunholtz DA, Edwards SJ, Lilford RJ. Are randomized clinical trials good for us (in the short term)? Evidence for a "trial effect. " J Clin Epidemiol. 2001;54(3):217-224.

21. Huang SS, MacRae J, Ross D, et al. Buttonhole versus stepladder cannulation for home hemodialysis: a multicenter, randomized, pilot trial. Clin J Am Soc. Nephrol. 2019;14(3):403410.

22. Van Eps CL, Jones $\mathrm{M}, \mathrm{Ng} \mathrm{T}$, et al. The impact of extended-hours home hemodialysis and buttonhole cannulation technique on hospitalization rates for septic events related to dialysis access. Hemodial Int. 2010;14(4):451-463.
23. O'Brien FJ, Kok HK, O'Kane C, et al. Arterio-venous fistula buttonhole cannulation technique: a retrospective analysis of infectious complications. Clin Kidney J. 2012;5(6):526-529.

24. Lok CE, Sontrop JM, Faratro R, Chan CT, Zimmerman DL. Frequent hemodialysis fistula infectious complications. Nephron Extra. 2014;4(3):159-167.

25. Nguyen DB, Shugart A, Lines C, et al. National Healthcare Safety Network (NHSN) dialysis event surveillance report for 2014. Clin J Am Soc Nephrol. 2017;12(7):1139-1146.

26. Taylor G, Gravel D, Johnston L, Embil J, Holton D, Paton S. Prospective surveillance for primary bloodstream infections occurring in Canadian hemodialysis units. Infect Control Hosp Epidemiol. 2002;23(12):716-720.

27. Nesrallah GE, Cuerden M, Wong JH, Pierratos A. Staphylococcus aureus bacteremia and buttonhole cannulation: longterm safety and efficacy of mupirocin prophylaxis. Clin J Am Soc Nephrol. 2010;5(6):1047-1053.

28. Boelaert JR, De Smedt RA, De Baere YA, et al. The influence of calcium mupirocin nasal ointment on the incidence of Staphylococcus aureus infections in haemodialysis patients. Nephrol Dial Transplant. 1989;4(4):278-281.

29. Hogue JS, Buttke P, Braun LE, Fairchok MP. Mupirocin resistance related to increasing mupirocin use in clinical isolates of methicillin-resistant Staphylococcus aureus in a pediatric population. J Clin Microbiol. 2010;48(7):2599-2600.

30. Upton A, Lang S, Heffernan H. Mupirocin and Staphylococcus aureus: a recent paradigm of emerging antibiotic resistance. J Antimicrob Chemother. 2003;51(3):613-617.

31. Boelaert JR, Van Landuyt HW, Gordts BZ, De Baere YA, Messer SA, Herwaldt LA. Nasal and cutaneous carriage of Staphylococcus aureus in hemodialysis patients: the effect of nasal mupirocin. Infect Control Hosp Epidemiol. 1996;17(12): 809-811.

32. Sato S, Shinzato T, Sakai N, et al. Deformity of buttonhole entry site causes higher frequency of vascular access-related infection. Contrib Nephrol. 2015;186:48-56.

33. Christensen LD, Skadborg MB, Mortensen AH, et al. Bacteriology of the buttonhole cannulation tract in hemodialysis patients: a prospective cohort study. Am J Kidney Dis. 2018;72(2):234-242.

34. Nielsen LH, Jensen-Fangel S, Benfield T, et al. Risk and prognosis of Staphylococcus aureus bacteremia among individuals with and without end-stage renal disease: a Danish, populationbased cohort study. BMC Infect Dis. 2015;15:6.

35. Imaizumi T, Hasegawa T, Nomura A, et al. Association between Staphylococcus aureus bacteremia and hospital mortality in hemodialysis patients with bloodstream infection: a multicenter cohort from Japanese tertiary care centers. Ther Apher Dial. 2017;21(4):354-360.

36. Høg BB, Korsgaard HB, Sönksen UW, et al. DANMAP 2016 use of antimicrobial agents and occurrence of antimicrobial resistance in bacteria from food animals, food and humans in Denmark. https://www.danmap.org/-/media/arkiv/projekt-sites/ danmap/danmap-reports/danmap-2016/danmap_2016_web. pdf?la=en. Accessed March 1, 2019.

37. Wertheim HF, Vos MC, Ott A, et al. Risk and outcome of nosocomial Staphylococcus aureus bacteraemia in nasal carriers versus non-carriers. Lancet. 2004;364(9435):703-705.

38. Fattom A, Matalon A, Buerkert J, Taylor K, Damaso S, Boutriau D. Efficacy profile of a bivalent Staphylococcus aureus glycoconjugated vaccine in adults on hemodialysis: phase III randomized study. Hum Vaccin Immunother. 2015;11(3):632-641. 